Pak. j. sci. ind. res. Ser. A: phy. sci. 2020 63A(3) 239-246

\title{
Review
}

\section{A Review on Nanocarriers for Cancer Targeted Drug Delivery}

\author{
Fariha Irfan*, Tahir Iqbal, Nafisa Malik and Mohsin Ijaz \\ Department of Physics, Faculty of Sciences, University of Gujrat, Hafiz Hayat Campus, \\ Gujrat, 50700, Pakistan
}

(received June 27, 2018; revised January 20, 2019; accepted January 24, 2019)

\begin{abstract}
Nanoparticles in the drugs are useful for the treatment of cancer due to their unique properties and can act as drug carriers in different ways. Unlike the traditional chemotherapy, the entrance of nanotechnology enabled wide applications in treatment of cancer. Although nanoparticles provides safe and effective drug delivery systems but the factor of toxicity still limits the utilisation of several nanoparticles. The properties of nanodrug carriers are controllable by various factors. The use of nanoparticles in cancer therapy by drug delivery and their advantages as been reviewed.
\end{abstract}

Keywords: drug carriers, passive targeting, active targeting, liposomes, metallic nanoparticles, dendrimers, polymeric nanoparticles

\section{Introduction}

Nanotechnology is a field that deals with the ultra-small particles which is stretched out to expansive region in pharmaceutical and other wide application due to its unique properties. Nanotechnology has many applications in field of medicine. Large number of people die every year due to cancer but nanotechnology is effectively playing an effective role to treat this disease (Hossen et al., 2018; Siegel et al., 2015). Nanoparticles have a large number of uses in diagnostic of diseases like cancer and their treatment. But these nanoparticles can damage the normal cell along with cancer cells due to their toxicity (Wang et al., 2012). Targeted treatment has developed as one way to deal with the absence of fineness of ordinary chemotherapeutic agents. As different types of chemical are used in chemotherapy to destroy the cancer cells but nanotechnology offers more suitable way to treat such diseases (Zhang et al., 2011). To target the cancer cells, nanoparticles can use two mechanisms which include passive targeting and active targeting (Cho et al., 2008). A proper type of nano carrier must be selected to adopt the best strategy to identify the different types of cancer cells (Hossen et al., 2018; Matsumura and Maeda, 1986).

Several type of nanoparticles like carbon nanotubes, polymeric nanoparticles, liposome, metallic and

*Author for correspondence;

E-mail: 18141710-014@uog.edu.pk magnetic nanoparticles are used for this purpose. Although nanoparticles have advantages but still they have several limitations which include instability in circulation, improper distribution of tissues, toxicity and poor oral bioavailability.Nanoparticles can target the drug to be effective for drug delivery.

Nanotechnology and targeted drug delivery. The best effect of nanotechnologies is drug delivery to treat the tumors. The therapeutic index of almost all drugs presently getting used may be advanced and it is possible if they may be greater successfully delivered to the organic targets via suitable nanotechnology application (Vasir et al., 2005; Sahoo and Labhasetwar, 2003). A few drugs which have formerly failed medical trials may additionally be re tested the usage of nano technological techniques. Some of barriers can be conquer with diverse novel packages of Nano drug delivery. As an example, some drugs aren't properly soluble which make it hard to manage therapeutic doses (Kipp, 2004; Rabinow, 2004). In central nervous system cancer, numerous medications experience issues in intersection the blood-brain boundary to target the cancer tumor. Nanocarriers can enter this barrier and have been appeared to increase the therapeutic concentrations of drug (Koziara et al., 2004; Steiniger et al., 2004). To minimize the toxicity of anticancer drug, direct the drug towards the target for enough time that it can take action at the target (Brannon-Peppas and Blanchette, 2012). 
Nanoparticles used as anti-cancer drug. Different nanoparticles are being used as drug carriers. We will talk about the properties of different nano drug carriers in this section (Fig. 1). All these nanocarriers have different properties and different potential to be used in drug delivery system and their effectiveness depends upon their and other properties. These all describe in Table 1.

Polymeric nanoparticles. Polymeric nanoparticles are strong colloidal particles arranged from bio-degradable polymers, for example, Chitosan and Collagen. Their size range from 50 to $300 \mathrm{~nm}$ which enable them to get into the vessels and then to be taken up by the cells, expanding the concentration of the drug at the target (Ochekpe et al., 2009). Most of such compounds are formed by the spontaneous self-assembly (Wang et al., 2012). By changing their physio-chemical properties

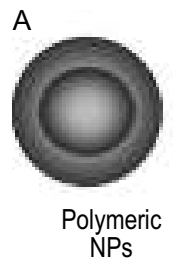

D

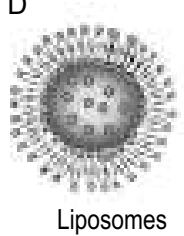

B

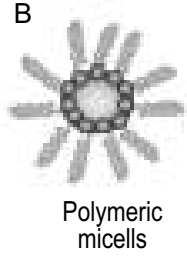

E

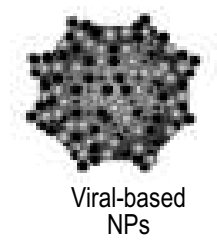

C

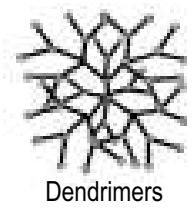

F

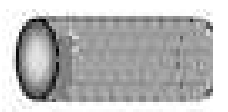

Carbon
Fig. 1. Different types of nanoparticles as drug carriers being used in drug delivery (Cho et al., 2008).

Table 1. (Different Nanocarriers approved and under trial for cancer treatment Kumari, et al., 2016

\begin{tabular}{ll}
\hline \hline Product & Disease and company \\
\hline CRLX101 & $\begin{array}{l}\text { Camptothecin Inc. (England) by Cerulean } \\
\text { pharma is in phase I/II to treat solid tumors. } \\
\text { CRLX101 }\end{array}$ \\
$\begin{array}{l}\text { Superparamagnetic iron-oxide by Advanced } \\
\text { magnetics USA is approved for MRI contrast } \\
\text { agent. }\end{array}$ \\
DepoCytTM & $\begin{array}{l}\text { Cytarabine for the treatment of } \\
\text { Lymphomatous meningitis by DeptoTech } \\
\text { corporation USA. } \\
\text { pDNA with p53 gene in phase I to treat solid } \\
\text { tumors (Synergene therapeutic USA). }\end{array}$ \\
\hline
\end{tabular}

and they can enhance the distinction to the target site of activity. (Fig. 2) shows the requirements for the design of polymeric nanoparticles for drug delivery system.

Polymeric micelles. These are formed by amphiphilic block copolymers. Their size is less than $100 \mathrm{~nm}$. Micelles are built with hydrophilic as well as hydrophobic part and are used for drug transport (Cagel et al., 2017; Qiao et al., 2010; Ochekpe et al., 2009). Due to their small size they can accumulate in pathogenic tissues. The drug amount, released by micelles is controllable by temperature and $\mathrm{pH}$ (Rapoport, 2007). Their target ability is not very good because of their low drug loading (Yamamoto et al., 2007).

Dendrimers. Dendrimers are highly branched synthetic polymers formed by macro molecules having less than $15 \mathrm{~nm}$ diameter of the inner core (Sanvicens and Marco, 2008). Their nanostructure consists of a focal core, functional groups and multiple interior layers (E Ruiz et al., 2014). Their size, shape and dimensions are controllable as they are formed by branched monomers. They have enough ability for the anticancer drug transport (Svenson and Tomalia, 2012). By changing the number of generation the surface charge density can be controlled.

Carbon based nanoparticles. Carbon nanotubes (CNTs) can penetrate into the cells. CNTs are of different types, multi-walled carbon nanotubes (MWNTs) and singlewalled carbon nanotubes (SWNTs) and it depends on the number of layers of the graphene sheet. They have unique size and shape and are very effective drug carriers

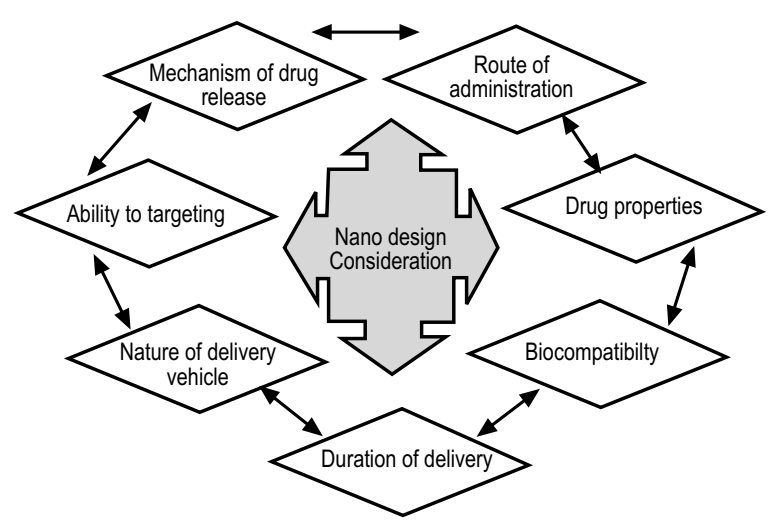

Fig. 2. Requirements to design a polymeric nanoparticle for the effective drug delivery system (Bennet and Kim 2014). 
(Chen et al., 2017; Kostarelos et al., 2007).Buckyball cluster belong to fullerenes. They are used for drug carriers and for detecting DNA. This nanoparticle shows insolubility in different solvents which causes toxicity. Their thermal and electronic properties made them good drug carriers.

Polymeric nanofibers. They are fibers having diameter from $1 \mathrm{~nm}-1 \mu \mathrm{m}$ and large surface area, tight pore size and low density. These properties can be changed by voltage. Nanofibers are composed of inorganic and organic materials. Nanofibers are used in medical for drug delivery but there are very few examples using these particles as anticancer drug.

Metallic nanoparticles. Metallic nanoparticles were discovered in 1971 and many of them are being used in medical. Gold and silver have electronic properties as well as optical properties (Goldman et al., 2004). Metallic nanoparticles of size between 10-100 nm are used as drug carriers. Other metallic nanoparticles are nickel, iron oxide, titanium and zinc oxide. Fig. 3 shows different shapes of gold nanoparticles.

Nano liposomes. Liposomes are closed colloidal structures that are self-assembled. They are formed by lipid bilayer which have spherical shape. An outer bilayer lipid covers a central space which is in aqueous form. Different kinds of anticancer drugs have been applied to this and it was done by using several preparation techniques. Liposomal formulations of the anthracyclines doxorubicin and daunorubicin are used to treat the breast cancer (Torchilin, 2005). Their size range from $20 \mathrm{~nm}-1 \mu \mathrm{m}$ (Samad et al., 2007). For proteins, nucleic acids and small molecules, Liposomes are mostly used delivery system. In 1961, Liposomes were very first nanoparticles used in medicine (Bangham and Horne, 1964). Nano Liposomes have the size range of 3-100 nm. Lipids used for the preparation of liposome are components of bilayer (Khosravi-Darani and Mozafari, 2010). The non-polar and polar regions of bilayer molecules allow the drug to set in lipid bilayer.
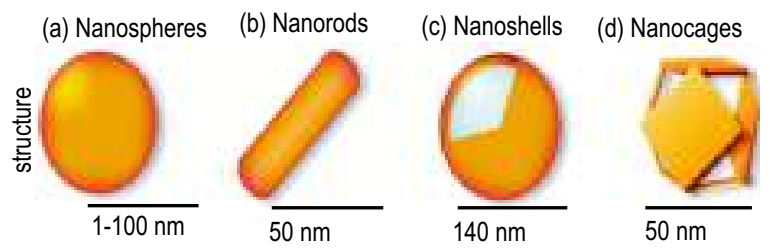

Fig. 3. Different shapes and sizes of gold nanoparticles(Hossen et al., 2018).
Nano Liposomes have wide use in medical and food industry. Chloroform is used as solvent for the formation of liposome but for therapeutic purpose, chloroform is not suitable. Nano Liposomes are good for low toxicity and biocompatibility. Biocompatible polymers can be coated on Liposomes such as polyethylene glycol. Liposome based drug delivery system is shown in (Fig. 4).

Passive and active targeting. Drug delivered have a tendency to uniformly scatter all through the body. However, tumor cells tent to take-up particles of a specific size to higher degree than solid cells because of the combination of broken tumor veins. This impact is affected by NP properties including magnitude, shape and surface charge. The unique properties of tumor vessels allow the macromolecules to accumulate in tissue. Micro environment surrounding tumor cells are also contributor to passive targeting and it is different from normal cells. The $\mathrm{pH}$ sensitive liposome is mostly stable at $\mathrm{pH}$ of 7.4.

The drug delivery system consists of binary conjugate depending on passive targeting face different intrinsic limitations to specificity. To overcome this situation, targeting ligands is included in drug conjugate (Sapra et al., 2005). The introduction of a large number of Liposomes drug carriers enhanced the number of drugs that may be the conjugate to the targeted NP. In active targeting, nanocarriers are guided to the tumor site (Hossen et al., 2018). Mechanism of passive and active targeting is shown in Fig. 5.

Requirements for effective drug. There must be a satisfactory medication focus in the body to into account

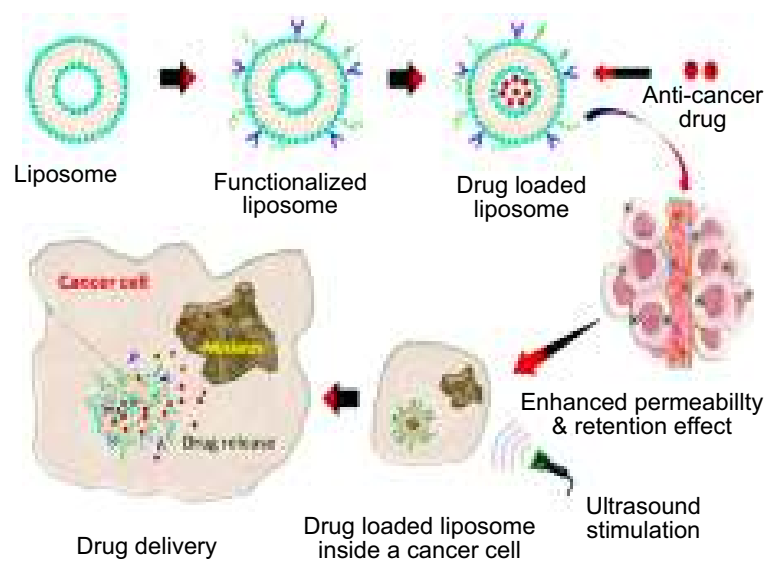

Fig. 4. Drug delivery system based on liposome against cancer (Hossen et al., 2018). 


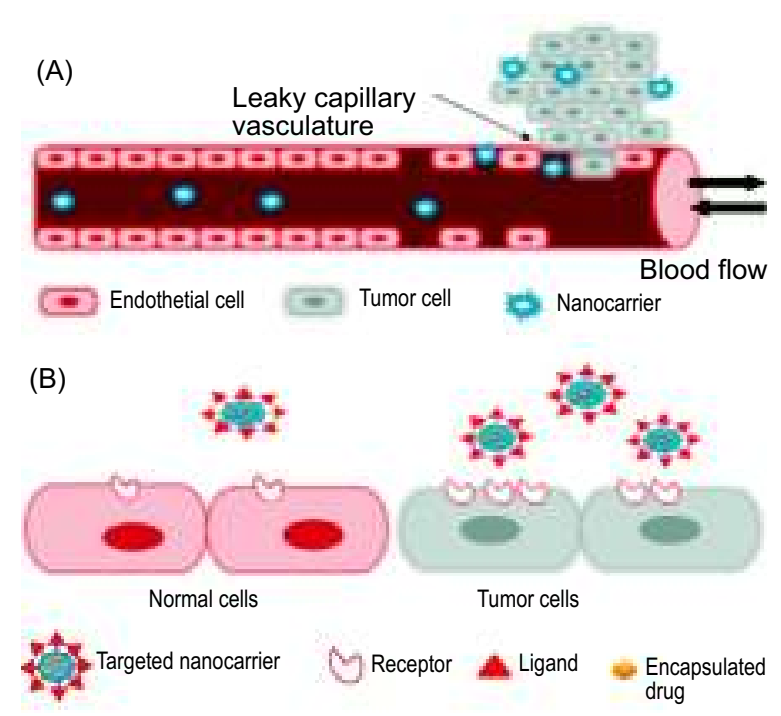

Fig. 5. Mechanism of (A) passive and (B) active targeting for tumors, (Kumari et al., 2016).

a successful measurement at tumor site. Target should be reluctant with the target's function. Monoclonal antibodies (used in diagnosis therapy) will be a fundamental part of new way for the treatment of cancer by nanotechnology.

To destroy the tumors, a fascinating methodology is through the use of antibodies (Gilboa, 2004; Pardoll, 2003). It is thought that by a proper immune response a body can remove little tumors. If it is possible to initiate such system against the tumors, they will be suitable to attack the cancer. Antigens with the solid core Nano beads forms a vaccine and the beads must have a size of 40-50 $\mathrm{nm}$ for effectiveness (Fifis et al., 2004). For deep tumors, energy sources can also harm the nearby tissues that are healthy tissues. So, by using the near infra-red absorbing gold nanoparticles, the tumors are heated. Thus an effective dose and methology is necessary for drug delivery.

\section{Strategies for cancer treatment by nanoparticles.}

- Metastatic cancer

- Non-targeted nanoparticles and targeted particles

Metastatic cancer is a clinical depiction for the cancer cells to spread from tumor too far off organs, building up new destinations for tumor. Separation of cancer cells from primary tumor in the blood, enables the cells to capture in organs, for example the lungs and skin, where they can increase in number (Chambers et al., 2002). These particles flowing in the blood have been appeared to significantly enhance drug bioavailability and collection in tumors through the upgraded permeability and retention effect. This effect permits the passive targeting to tumors. The idea of targeted treatment appeared in 1970 when antibodies were developed (Schrama et al., 2006). Targeted and untargeted nanoparticles drug delivery is shown in Fig. 6.

Types of stimulus to release the drug. Stimuli are of two types, one is exogenous and the other is endogenous. Signals, which include magnetic field, change in temperature and electric field are exogenous stimulus. On the other hand, endogenous stimuli include redox reactions, temperature and change in $\mathrm{pH}$. Endogenous signal is produced from inside the body (Mura et al., 2013).

Advantages of nanodrug carriers. Nanotechnology is fast growing field that uses the nanodrug carriers for the medication purposes which describe in Table 2 . Nanoparticles are being used in cancer therapy due to their unique properties. These unique properties allow them to be use as drug carriers. The most common nanodrug carriers are carbon based NPs, Liposomes and metallic NPs. NP drug delivery provides many advantages as drug carriers:

- Increase drug efficacy

- Low drug toxicity

- Improved therapeutic index

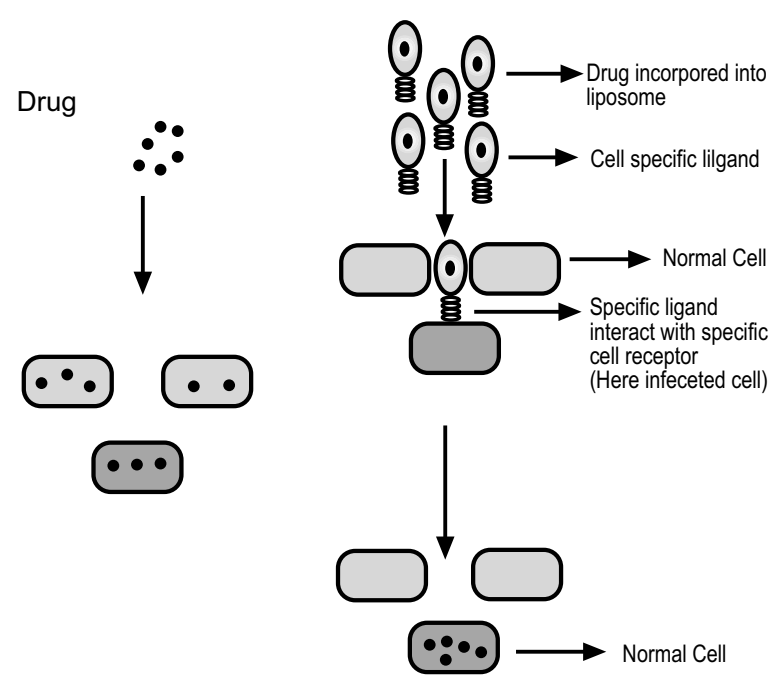

Fig. 6. Targeted and untargeted nanoparticles drug delivery (Prabhu et al., 2011). 
Table 2. Different endogenous stimuli

Enzyme stimulus

For biochemical reactions, enzymes are catalysts and are very important for the cells to perform their functions. Thus for drug delivery, enzymes are an important target. For intracellular drug release, the enzyme-triggered strategy is not applicable. Because the healthy cells and cancer cells have almost the same intracellular concentrations of enzymes (Andresen, et al., 2010).

The $\mathrm{pH}$ responsive stimulus

Redox sensitive A highly effective antioxidant, stimulus
Due to the enhanced glycolysis, energy is primarily produced by the tumor cells and this process is followed by the fermentation of lactic acid. This causes to lower the $\mathrm{pH}$ level in cancer cells. Different organs have different $\mathrm{pH}$ level. In tumors, extracellular $\mathrm{pH}$ has acidic environment as compared to the intracellular $\mathrm{pH}$. That is why $\mathrm{pH}$ responsive stimulus is very effective. Poor blood flow causes the extracellular $\mathrm{pH}$ to be acidic and its range is 6-7. Nanocarriers, which are $\mathrm{pH}$ sensitive, stores the drug and release at $\mathrm{pH}$ trigger point (Vander, et al., 2009; Gerweck, and Seetharaman, 1996; Vaupel, et al., 1989; Engin et al., 1995).

Glutathione Sulfhydryl (GSH), composed of three amino acids is present in mammalian tissues. It controls reductive micro-environment. A normal cell has four times higher concentration of GSH than a tumor site. Disulfide bonds can be reduced by GSH in Nanocarriers. This reduction can cause to release the drug (Gamcsik et al., 2012)
Nanoparticles as anticancer agents reduces the chemo resistance to drug action which increases their effect toward the cancer cell and decreases the effect for a normal and healthy tissue (Serpe, 2006).

Limitations of EPR effect. Nanocarriers drug delivery intervened by EPR effect (Enhanced permeability and retention) are thought to be successful against cancer (Torchilin, 2011). Most of the tumors have defective construction of blood vessels and shows a very good vascular permeability to supply enough oxygen to the tissues of tumor. Macromolecules, which are larger than $40 \mathrm{k}$ Da extravasated by EPR effect from the vessels of tumor. Anticancer drug delivery based on EPR effect has shown some of its effects in targeted drug delivery of chemotherapeutic agents. But it may face some problems to deliver the drug.

Drawbacks as drug carriers. Nanotechnology has played an effective role in the drug delivery system.Silver nanoparticles of size $100 \mathrm{~nm}$ have almost 20-1500 silver atoms, which can effectively treat the wounds (Lyczak and Schechter, 2005). Across the cellular barrier drug can be delivered by dendrimers. Nanoparticles can also deliver the drug for the treatment of tuberculosis.

On the other hand, these drug carriers have drawbacks which limit the use of drug carriers in medical field. Toxicity is the major drawback which may leads to different problems in medication. Nanoparticles must be able to produce drug resistance in case of any delay during the release of drug (Cairns et al., 2006).

Different nanocarriers shows different status of toxicity, given as;

(i) In different cell lines, in-vitro growth can be affected by cationic liposomes

(ii) CNT based Nanocarriers showed no toxicity in interaction with $\mathrm{CHO}$

(iii) Gold nanoparticles showed toxicity in some investigations, while some investigations showed that gold nanoparticles are not toxic

(iv) Polymeric micelles showed less toxicity in research

(v) Cytotoxicity is size dependent in silica nanoparticles. Larger particles showed less toxicity.

Future perspectives. Nanoparticles are being effectively used in medicine (drug delivery system). They have enough potential to treat AIDS. Protein delivery, antibiotics, vaccine and gene therapy are some other 
applications in which nanoparticles can play an effective role but still the major challenge is toxicity.

\section{Conclusion}

The development of nanoparticles for the targeted drug delivery has improved the effectiveness of drugs against cancer. Due to these nanoparticles the limitations of traditional chemotherapy can be overcome. Drug loaded nanocarriers can hit the target passively or actively. The challenges in nanoparticles as drug carriers are their loading efficiency and toxicity. It has two solutions, one is to reduce the toxicity and the other is to develop more nanocarriers which offer less toxicity. Different factors make the drug, effective for anticancer tumors. Application of nanotechnology for cancer therapy has shown good results and predicting the future of nanoparticles as anticancer drug is not simple as it is developing very fast. Research is required for the effectiveness of nanoparticles as drug without toxicity.

\section{Acknowledgment}

A worthy support of my dearest parents, respected Dr. Tahir Iqbal Awan (Assistant Professor, Department of Physics, University of Gujrat) is greatly acknowledged.

Conflict of Interest. The authors declare no conflict of interest.

\section{References}

Andresen, T.L., Thompson, D.H., Kaasgaard, T. 2010. Enzyme-triggered nanomedicine: drug release strategies in cancer therapy (invited review). Molecular Membrane Biology, 27: 353-363.

Bangham, A.D., Horne, R. 1964. Negative staining of phospholipids and their structural modification by surface-active agents as observed in the electron microscope. Journal of Molecular Biology, 8: 660IN610.

Bennet, D., Kim, S. 2014. Polymer nanoparticles for smart drug delivery. In: Application of Nanotechnology in Drug Delivery. Editor Ali Demir Seger pp.257-310, Publisher InTech.

Brannon-Peppas, L., Blanchette, J.O. 2012. Nanoparticle and targeted systems for cancer therapy. Advanced Drug Delivery Reviews, 64: 206-212.

Cagel, M., Tesan, F.C., Bernabeu, E., Salgueiro, M.J., Zubillaga, M.B., Moretton, M.A., Chiappetta, D.A. 2017. Polymeric mixed micelles as nanomedicines: achievements and perspectives. European Journal of Pharmaceutics and Biopharmaceutics, 113: 211228.

Cairns, R., Papandreou, I., Denko, N. 2006. Overcoming physiologic barriers to cancer treatment by molecularly targeting the tumor micro environment. Molecular Cancer Research, 4: 61-70.

Chambers, A.F., Groom, A.C., MacDonald, I.C. 2002. Metastasis: dissemination and growth of cancer cells in metastatic sites. Nature Reviews Cancer, 2: 563 .

Chen, Z., Zhang, A., Wang, X., Zhu, J., Fan, Y., Yu, H., Yang, Z. 2017. The advances of carbon nanotubes in cancer diagnostics and therapeutics. Journal of Nanomaterials, 2017.

Cho, K., Wang, X., Nie, S., Shin, D.M. 2008. Therapeutic nanoparticles for drug delivery in cancer. Clinical Cancer Research, 14: 1310-1316.

Engin, K., Leeper, D.B., Cater, J.R., Thistlethwaite, A.J., Tupchong, L., McFarlane, J.D. 1995. Extracellular $\mathrm{pH}$ distribution in human tumours. International Journal of Hyperthermia, 11: 211216.

Fifis, T., Gamvrellis, A., Crimeen-Irwin, B., Pietersz, G.A., Li, J., Mottram, P.L., Plebanski, M. 2004. Size-dependent immunogenicity: therapeutic and protective properties of nano-vaccines against tumors. The Journal of Immunology, 173: 31483154.

Gamcsik, M.P., Kasibhatla, M.S., Teeter, S.D., Colvin, O.M. 2012. Glutathione levels in human tumors. Biomarkers, 17: 671-691.

Gerweck, L.E., Seetharaman, K. 1996. Cellular pH gradient in tumer versus normal tissue: potential exploitation for the treatment of cancer. Cancer Research, 56: 1194-1198.

Gilboa, E. 2004. The promise of cancer vaccines. Nature Reviews Cancer, 4: 401.

Goldman, E.R., Clapp, A.R., Anderson, G.P., Uyeda, H.T., Mauro, J.M., Medintz, I.L., Mattoussi, H. 2004. Multiplexed toxin analysis using four colors of quantum dot fluororeagents. Analytical Chemistry, 76: 684-688.

Hossen, S., Hossain, M.K., Basher, M.K., Mia, M.N.H., Rahman, M.T., Uddin, M.J. 2018. Smart nanocarrier-based drug delivery systems for cancer therapy and toxicity studies: A review. Journal of Advanced Research, 15: 1-18. 
Khosravi-Darani, K., Mozafari, M. 2010. Nanoliposome potentials in nanotherapy: A concise overview. International Journal of Nanoscience and Nanotechnology, 6: 3-13.

Kipp, J. 2004. The role of solid nanoparticle technology in the parenteral delivery of poorly water-soluble drugs. International Journal of Pharmaceutics, 284: 109-122.

Kostarelos, K., Lacerda, L., Pastorin, G., Wu, W., Wieckowski, S., Luangsivilay, J., Muller, S. 2007. Cellular uptake of functionalized carbon nanotubes is independent of functional group and cell type. Nature Nanotechnology, 2: 108.

Koziara, J.M., Lockman, P.R., Allen, D.D., Mumper, R.J. 2004. Paclitaxel nanoparticles for the potential treatment of brain tumors. Journal of Controlled Release, 99: 259-269.

Kumari, P., Ghosh, B., Biswas, S. 2016. Nanocarriers for cancer-targeted drug delivery. Journal of Drug Targeting, 24: 179-191.

Lyczak, J.B., Schechter, P.J. 2005. Nano crystalline silver inhibits antibiotic-, antiseptic-resistant bacteria. Clinical Pharmacology \& Therapeutics, 77: P60-P60.

Matsumura, Y., Maeda, H. 1986. A new concept for macromolecular therapeutics in cancer chemotherapy: mechanism of tumoritropic accumulation of proteins and the antitumor agent smancs. Cancer Research, 46: 6387-6392.

Mura, S., Nicolas, J., Couvreur, P. 2013. Stimuliresponsive nanocarriers for drug delivery. Nature Materials, 12: 991.

Ochekpe, N.A., Olorunfemi, P.O., Ngwuluka, N.C. 2009. Nanotechnology and drug delivery part 2 : nanostructures for drug delivery. Tropical Journal of Pharmaceutical Research, 8: 275-287.

Pardoll, D. 2003. Does the immune system see tumors as foreign or self. Annual Review of Immunology, 21: 807-839.

Prabhu, V., Uzzaman, S., Grace, V.M.B., Guruvayoorappan, C. 2011. Nanoparticles in drug delivery and cancer therapy: the giant rats tail. Journal of Cancer Therapy, 2: 325-334.

Qiao, W., Wang, B., Wang, Y., Yang, L., Zhang, Y., Shao, P. 2010. Cancer therapy based on nanomaterials and nanocarrier systems. Journal of Nanomaterials, 2010; https://doi .org/101155/2010/ 796303.

Rabinow, B.E. 2004. Nanosuspensions in drug delivery. Nature Reviews Drug Discovery, 3: 785.

Rapoport, N. 2007. Physical stimuli-responsive polymeric micelles for anti-cancer drug delivery. Progress in Polymer Science, 32: 962-990.

Ruiz, M.E., Gantner, M.E., Talevi, A. 2014. Applications of nanosystems to anticancer drug therapy (Part II. dendrimers, micelles, lipid-based nanosystems). Recent Patents on Anti-Cancer Drug Discovery, 9: 99-128.

Sahoo, S. K., Labhasetwar, V. 2003. Nanotech approaches to drug delivery and imaging. Drug Discovery Today, 8: 1112-1120.

Samad, A., Sultana, Y., Aqil, M. 2007. Liposomal drug delivery systems: an update review. Current Drug Delivery, 4: 297-305.

Sanvicens, N., Marco, M.P. 2008. Multifunctional nanoparticles-properties and prospects for their use in human medicine. Trends in Biotechnology, 26: 425-433.

Sapra, P., Tyagi, P., Allen, T. M. 2005. Ligand-targeted liposomes for cancer treatment. Current Drug Delivery, 2: 369-381.

Schrama, D., Reisfeld, R.A., Becker, J.C. 2006. Antibody targeted drugs as cancer therapeutics. Nature Reviews Drug Discovery, 5: 147.

Serpe, L., Braet, L., Soon, T.F., Kelly, D., Larson, Simon, P.R. 2006. Conventional chemotherapeutic drug nanoparticles for cancer treatment. NanoTechnologies for the Life Sciences, 3: 489.

Siegel, R.L., Miller, K.D., Jemal, A. 2015. Cancer statistics, 2015. CA: A Cancer Journal for Clinicians, 65: 5-29.

Steiniger, S.C., Kreuter, J., Khalansky, A.S., Skidan, I.N., Bobruskin, A.I., Smirnova, Z.S., Geiger, K.D. 2004. Chemotherapy of glioblastoma in rats using doxorubicin-loaded nanoparticles. International Journal of Cancer, 109: 759-767.

Svenson, S., Tomalia, D.A. 2012. Dendrimers in biomedical applications-reflections on the field. Advanced Drug Delivery Reviews, 64: 102-115.

Torchilin, V.P. 2005. Recent advances with liposomes as pharmaceutical carriers. Nature Reviews Drug Discovery, 4: 145.

Torchilin, V. 2011. Tumor delivery of macromolecular drugs based on the EPR effect. Advanced Drug Delivery Reviews, 63: 131-135.

Vander Heiden, M.G., Cantley, L.C., Thompson, C.B. 2009V. Understanding the Warburg effect: the metabolic requirements of cell proliferation. Science, 324: 1029-1033.

Vasir, J.K., Reddy, M.K., Labhasetwar, V.D. 2005. Nanosystems in drug targeting: opportunities and 
challenges. Current Nanoscience, 1: 47-64.

Vaupel, P., Kallinowski, F., Okunieff, P. 1989. Blood flow, oxygen and nutrient supply, and metabolic microenvironment of human tumors: a review. Cancer Research, 49: 6449-6465.

Wang, A.Z., Langer, R., Farokhzad, O.C. 2012. Nanoparticle delivery of cancer drugs. Annual Review of Medicine, 63: 185-198.

Yamamoto, T., Yokoyama, M., Opanasopit, P., Hayama,
A., Kawano, K., Maitani, Y. 2007. What are determining factors for stable drug incorporation into polymeric micelle carriers? Consideration on physical and chemical characters of the micelle inner core. Journal of Controlled Release, 123: 11-18.

Zhang, W., Zhang, Z., Zhang, Y. 2011. The application of carbon nanotubes in target drug delivery systems for cancer therapies. Nanoscale Research Letters, 6: 555 . 